\title{
Computation of Topological Indices of Dutch Windmill Graph
}

\author{
M. R. Rajesh Kanna1, R. Pradeep Kumar'2, R. Jagadeesh ${ }^{3}$ \\ ${ }^{1}$ Post Graduate Department of Mathematics, Maharani's Science College for Women, \\ Mysore, India \\ ${ }^{2}$ Department of Mathematics, The National Institute of Engineering, Mysore, India \\ ${ }^{3}$ Research and Development Centre, Bharathiar University, Coimbatore, India \\ Email:mr.rajeshkanna@gmail.com,pradeepr.mysore@gmail.com,jagadeeshr1978@gmail.com
}

Received 11 January 2016; accepted 5 April 2016; published 8 April 2016

Copyright (C) 2016 by authors and Scientific Research Publishing Inc.

This work is licensed under the Creative Commons Attribution International License (CC BY).

http://creativecommons.org/licenses/by/4.0/

c) (i) Open Access

\begin{abstract}
In this paper, we compute Atom-bond connectivity index, Fourth atom-bond connectivity index, Sum connectivity index, Randic connectivity index, Geometric-arithmetic connectivity index and Fifth geometric-arithmetic connectivity index of Dutch windmill graph.
\end{abstract}

\section{Keywords}

$A B C$ Index, $A B C_{4}$ Index, Sum Connectivity Index, Randic Connectivity Index, $G A$ Index, $G A_{5}$ Index

\section{Introduction}

The Dutch windmill graph is denoted by $D_{n}^{(m)}$ and it is the graph obtained by taking $m$ copies of the cycle $C_{n}$ with a vertex in common. The Dutch windmill graph is also called as friendship graph if $n=3$. i.e., friendship graph is the graph obtained by taking $m$ copies of the cycle $C_{3}$ with a vertex in common. Dutch windmill graph $D_{n}^{(m)}$ contains $(n-1) m+1$ vertices and $m n$ edges as shown in the Figures $1-3$.

All graphs considered in this paper are finite, connected, loop less and without multiple edges. Let $G=(V, E)$ be a graph with $n$ vertices and $m$ edges. The degree of a vertex $u \in V(G)$ is denoted by $d_{u}$ and is the number of vertices that are adjacent to $u$. The edge connecting the vertices $u$ and $v$ is denoted by $u v$. Using these terminologies, certain topological indices are defined in the following manner.

Topological indices are numerical parameters of a graph which characterize its topology and are usually graph invariants.

The atom-bond connectivity index, $A B C$ index was one of the degree-based molecular descripters, which was introduced by Estrada et al. [1] in late 1990's. Some upper bounds for the atom-bond connectivity index of 


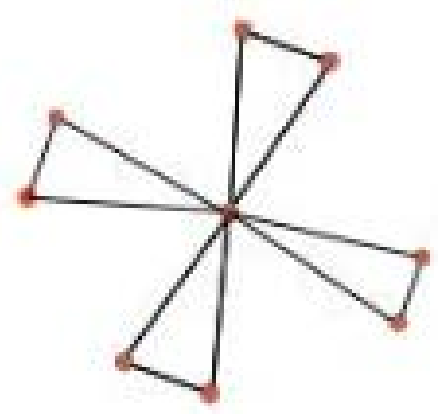

Figure 1. $D_{3}^{(4)}$.

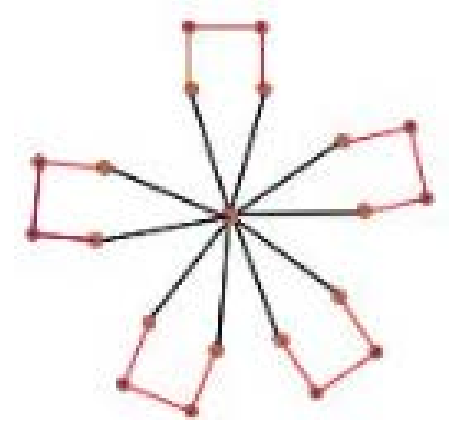

Figure 2. $D_{5}^{(5)}$.

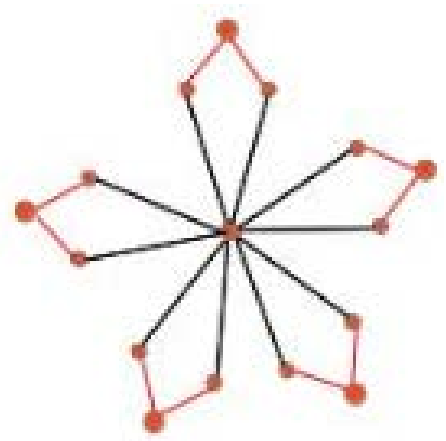

Figure 3. $D_{4}^{(5)}$.

graphs can be found in [2], The atom-bond connectivity index of chemical bicyclic graphs and connected graphs can be seen in [3] [4]. For further results on $A B C$ index of trees, see the papers [5]-[8] and the references cited there in.

Definition 1.1. Let $G=(V, E)$ be a molecular graph and $d_{u}$ is the degree of the vertex $u$, then $A B C$ index of $G$ is defined as, $A B C(G)=\sum_{u v \in E} \sqrt{\frac{d_{u}+d_{v}-2}{d_{u} d_{v}}}$.

The fourth atom bond connectivity index, $A B C_{4}(G)$ index was introduced by M. Ghorbani et al. [9] in 2010. Further studies on $A B C_{4}(G)$ index can be found in [10] [11].

Definition 1.2. Let $G$ be a graph, then its fourth $A B C$ index is defined as, $A B C_{4}(G)=\sum_{u v \in E(G)} \sqrt{\frac{S_{u}+S_{v}-2}{S_{u} S_{v}}}$, where $S_{u}$ is sum of the degrees of all neighbours of vertex $u$ in G. In other words, $S_{u}=\sum_{u v \in E(G)} d_{v}$, Similarly for $S_{v}$. 
The first and oldest degree based topological index was Randic index [12] denoted by $\chi(G)$ and was introduced by Milan Randic in 1975 .

Definition 1.3. For the graph $G$ Randic index is defined as, $\chi(G)=\sum_{u v \in E(G)} \frac{1}{\sqrt{d_{u} d_{v}}}$.

Sum connectivity index belongs to a family of Randic like indices. It was introduced by Zhou and Trinajstic [13]. Further studies on Sum connectivity index can be found in [14] [15].

Definition 1.4. For a simple connected graph $G$, its sum connectivity index $S(G)$ is defined as, $S(G)=\sum_{u v \in E(G)} \frac{1}{\sqrt{d_{u}+d_{v}}}$.

The Geometric-arithmetic index, $G A(G)$ index of a graph $G$ was introduced by D. Vukicevic et al. [16]. Further studies on GA index can be found in [17]-[19].

Definition 1.5. Let $G$ be a graph and $e=u v$ be an edge of $G$ then, $G A(G)=\sum_{u v \in E(G)} \frac{2 \sqrt{d_{u} d_{v}}}{d_{u} d_{v}}$.

The fifth Geometric-arithmetic index, $G A_{5}(G)$ was introduced by A.Graovac et al. [20] in 2011.

Definition 1.6. For a Graph $G$, the fifth Geometric-arithmetic index is defined as $G A_{5}(G)=\sum_{u v \in E(G)} \frac{2 \sqrt{S_{u} S_{v}}}{S_{u}+S_{v}}$,

Where $S_{u}$ is the sum of the degrees of all neighbors of the vertex $u$ in $G$, similarly for $S_{v}$.

\section{Main Results}

Theorem 2.1. The Atom bond connectivity index of Dutch windmill graph is $A B C\left(D_{n}^{(m)}\right)=\frac{m n}{\sqrt{2}}$.

Proof. Consider the Dutch windmill graph $D_{n}^{(m)}$. We partition the edges of $D_{n}^{(m)}$ into edges of the type $E_{\left(d_{u}, d_{v}\right)}$ where $u v$ is an edge. In $D_{n}^{(m)}$ we get edges of the type $E_{(2,2)}$ and $E_{(2 n, 2)}$. Edges of the type $E_{(2,2)}$ and $E_{(2 n, 2)}$ are colored in red and black respectively as shown in the figure [18]. The number of edges of these types are given in the Table 1 .

We know that $A B C(G)=\sum_{u v \in E(G)} \sqrt{\frac{d_{u}+d_{v}-2}{d_{u} d_{v}}}$

i.e., $\quad A B C\left(D_{n}^{(m)}\right)=\left|E_{(2,2)}\right| \sum_{u v \in E_{(2,2)}(G)} \sqrt{\frac{d_{u}+d_{v}-2}{d_{u} d_{v}}}+\left|E_{(2 m, 2)}\right| \sum_{u v \in E_{(2 m, 2)}(G)} \sqrt{\frac{d_{u}+d_{v}-2}{d_{u} d_{v}}}$

$$
A B C\left(D_{n}^{(m)}\right)=(n-2) m \sqrt{\frac{2+2-2}{2 \cdot 2}}+2 m \sqrt{\frac{2 m+2-2}{2 m \cdot 2}}
$$

[From Table 1 and Figure 4]

$$
=(n-2) m \frac{1}{\sqrt{2}}+2 m \frac{1}{\sqrt{2}}=\frac{m n}{\sqrt{2}} .
$$

Theorem 2.2. The Randic Index of Dutch windmill graph is $\chi\left(D_{n}^{(m)}\right)=\frac{(n-2) m+2 \sqrt{m}}{2}$

Proof. We know that $\chi(G)=\sum_{u v \in E(G)} \frac{1}{\sqrt{d_{u} d_{v}}}$

Table 1. Edge partition based on degrees of end vertices of each edge.

\begin{tabular}{cc}
\hline Edges of the type $E_{\left(d_{u}, d_{v}\right)}$ & Number of edges \\
$E_{(2,2)}$ & $(\mathrm{n}-2) \mathrm{m}$ \\
$E_{(2 \mathrm{~m}, 2)}$ & $2 \mathrm{~m}$ \\
\hline
\end{tabular}




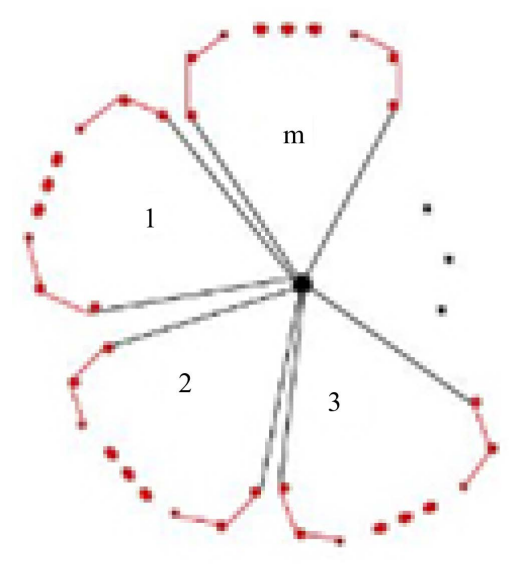

Figure 4. $D_{n}^{(m)}$.

$$
\begin{aligned}
\text { i.e., } & \chi\left(D_{n}^{(m)}\right)=\left|E_{(2,2)}\right| \sum_{u v \in E_{(2,2)}(G)} \frac{1}{\sqrt{d_{u} d_{v}}}+\left|E_{(2 m, 2)}\right| \sum_{u v \in E_{(2 m, 2)}(G)} \frac{1}{\sqrt{d_{u} d_{v}}} \\
= & (n-2) m \frac{1}{\sqrt{2 \cdot 2}}+2 m \frac{1}{\sqrt{2 m \cdot 2}} \text { [From Table } 1 \text { and Figure 4] } \\
= & \frac{(n-2) m+2 \sqrt{m}}{2} .
\end{aligned}
$$

Theorem 2.3. The Geometric-arithmetic index (GA) of Dutch windmill graph is

$$
G A\left(D_{n}^{(m)}\right)=\frac{m(m n-2 m+n-2+4 \sqrt{m})}{m+1} .
$$

Proof. We know that $G A(G)=\sum_{u v \in E(G)} \frac{2 \sqrt{d_{u} d_{v}}}{\left(d_{u}+d_{v}\right)}$

$$
G A\left(D_{n}^{(m)}\right)=\left|E_{(2,2)}\right| \sum_{u v \in E_{(2,2)}(G)} \frac{2 \sqrt{d_{u} d_{v}}}{\left(d_{u}+d_{v}\right)}+\left|E_{(2 m, 2)}\right| \sum_{u v \in E_{(2 m, 2)}(G)} \frac{2 \sqrt{d_{u} d_{v}}}{\left(d_{u}+d_{v}\right)}
$$

$=(n-2) m \frac{2 \sqrt{2 \cdot 2}}{2+2}+2 m \frac{2 \sqrt{2 m \cdot 2}}{2 m+2} \quad$ [From Table 1 and Figure 4]

$=\frac{m(m n-2 m+n-2+4 \sqrt{m})}{m+1}$.

Theorem 2.4. The Sum connectivity index $S(G)$ of Dutch windmill graph is $S(G)=\frac{(n-2) m}{2}+\frac{m \sqrt{2}}{\sqrt{m+1}}$.

Proof. We know that $S(G)=\sum_{u v \in E(G)} \frac{1}{\sqrt{d_{u}+d_{v}}}$

i.e., $S\left(D_{n}^{(m)}\right)=\left|E_{(2,2)}\right| \sum_{u v \in E_{(2,2)}(G)} \frac{1}{\sqrt{d_{u}+d_{v}}}+\left|E_{(2 m, 2)}\right| \sum_{u v \in E_{(2 m, 2)}(G)} \frac{1}{\sqrt{d_{u}+d_{v}}}$

$=(n-2) m \frac{1}{\sqrt{2+2}}+2 m \frac{1}{\sqrt{2 m+2}}$ [From Table 1 and Figure 4 ]

$=\frac{(n-2) m}{2}+\frac{m \sqrt{2}}{\sqrt{m+1}}$.

Theorem 2.5. The fourth atom bond connectivity index of Dutch windmill graph is 


$$
A B C_{4}\left(D_{m}^{(n)}\right)= \begin{cases}\frac{m}{\sqrt{m+1}}[(n-4) \sqrt{6(m+1)}+\sqrt{m+2}+\sqrt{3}] & \text { if } n \geq 4 \\ \frac{m}{\sqrt{m+1}}\left[\sqrt{\frac{2 m+1}{2(m+1)}}+\sqrt{3}\right] & \text { if } n=3\end{cases}
$$

Proof. Any Dutch windmill graph $D_{n}^{(m)}$ contains $(n-1) m+1$ vertices and $m n$ edges. Let $d_{u}$ denote the degree of the vertex $u$. We partition the edges of $D_{n}^{(m)}$ into edges of the type $E_{\left(S_{u}, S_{v}\right)}^{*}$ where $u v$ is an edge and $S_{u}$ is the sum of the degrees of all neighbours of vertex u in G. In other words, $\stackrel{\left.S_{u}, v_{v}\right)}{=}=\sum_{u v \in E(G)} d_{v}$, Similarly for $S_{v}$.

Case (1) If $n \geq 4$ : In $D_{n}^{(m)}$ we get edges of the type $E_{(4,4)}^{*}, E_{(4,2 m+2)}^{*}$ and $E_{(2 m+2,4 m)}^{*}$. Edges of the type $E_{(4,4)}^{*}, E_{(4,2 m+2)}^{*}$ and $E_{(2 m+2,4 m)}^{*}$ are colored in red, green and black respectively as shown in the figure [1]. The number of edges of these types are given in the Table 2.

We know that $A B C_{4}(G)=\sum_{u v \in E(G)} \sqrt{\frac{S_{u}+S_{v}-2}{S_{u} S_{v}}}$ i.e.,

$$
A B C_{4}\left(D_{n}^{(m)}\right)=\left|E_{(4,4)}^{*}\right| \sum_{u v \in E_{(4,4)}^{*}(G)} \sqrt{\frac{S_{u}+S_{v}-2}{S_{u} S_{v}}}+\left|E_{(4,2 m+2)}^{*}\right| \sum_{u v \in E_{(4,2 m+2)}^{*}(G)} \sqrt{\frac{S_{u}+S_{v}-2}{S_{u} S_{v}}}
$$

$$
+\left|E_{(2 m+2,4 m)}^{*}\right| \sum_{u v \in E_{(2 m+2,4 m)}^{*}(G)} \sqrt{\frac{S_{u}+S_{v}-2}{S_{u} S_{v}}}
$$

[From Table 2 and Figure 5]

$$
\begin{gathered}
=(n-4) m \sqrt{\frac{4+4-2}{4 \cdot 4}}+2 m \sqrt{\frac{4+2 m+2-2}{4(2 m+2)}}+2 m \sqrt{\frac{2 m+2+4 m-2}{4 m(2 m+2)}} \\
=\frac{(n-4) m \sqrt{6}}{4}+m \sqrt{\frac{m+2}{m+1}}+m \sqrt{\frac{3}{m+1}}
\end{gathered}
$$

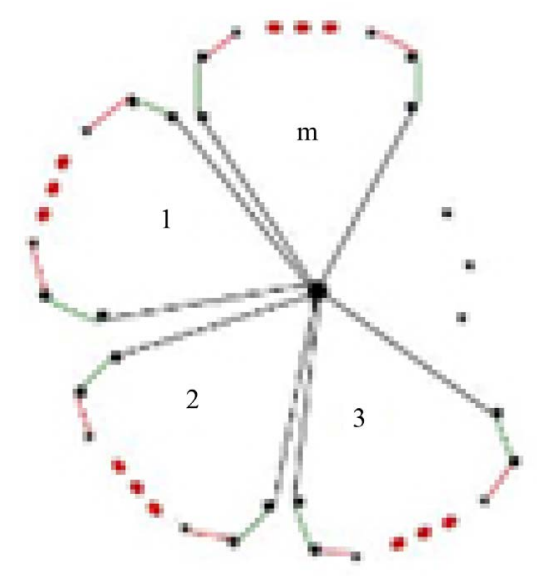

Figure 5. $D_{n}^{(m)}$.

Table 2. Edge partition based on degree sum of neighbors of end vertices of each edge.

\begin{tabular}{cc}
\hline Edges of the type & Number of edges \\
\hline$E_{(4,4)}^{*}$ & $(\mathrm{n}-4) \mathrm{m}$ \\
$E_{(4,2 m+2)}^{*}$ & $2 \mathrm{~m}$ \\
$E_{(2 \mathrm{~m}+2,4 \mathrm{~m})}^{*}$ & $2 \mathrm{~m}$ \\
\hline
\end{tabular}




$$
=\frac{m}{\sqrt{m+1}}[(n-4) \sqrt{6(m+1)}+\sqrt{m+2}+\sqrt{3}] .
$$

Case (2) If $n=3$ : In $D_{3}^{(m)}$ we get edges of the type $E_{(2 m+2,2 m+2)}^{*}$ and $E_{(2 m+2,4 m)}^{*}$. The number of edges of these types are given in the Table 3.

We know that $A B C_{4}(G)=\sum_{u v \in E(G)} \sqrt{\frac{S_{u}+S_{v}-2}{S_{u} S_{v}}}$

i.e.,

$$
\begin{aligned}
A B C_{4}\left(D_{n}^{(m)}\right)= & \left|E_{(2 m+2,2 m+2)}^{*}\right| \sum_{u v \in E_{(2 m+2,2 m+2)}^{*}(G)} \sqrt{\frac{S_{u}+S_{v}-2}{S_{u} S_{v}}} \\
& +\left|E_{(2 m+2,4 m)}^{*}\right| \sum_{u v \in E_{(2 m+2,4 m)}^{*}(G)} \sqrt{\frac{S_{u}+S_{v}-2}{S_{u} S_{v}}} \\
= & m \sqrt{\frac{2 m+2+2 m+2-2}{(2 m+2)(2 m+2)}}+2 m \sqrt{\frac{2 m+2+4 m-2}{(2 m+2) 4 m}} \\
= & m \frac{\sqrt{2(2 m+1)}}{2(m+1)}+m \sqrt{\frac{3}{m+1}}=\frac{m}{\sqrt{m+1}}\left[\sqrt{\frac{2 m+1}{2(m+1)}}+\sqrt{3}\right] .
\end{aligned}
$$

Theorem 2.6. The fifth Geometric-arithmetic index $\left(G A_{5}\right)$ of Dutch windmill graph is

$$
G A_{5}\left(D_{n}^{(m)}\right)= \begin{cases}(n-4) m+\frac{4 m \sqrt{2(m+1)}}{3}+\frac{2 m \sqrt{2 m(m+1)}}{3 m+1} & \text { if } n \geq 4 \\ m\left[1+\frac{4 \sqrt{2 m(m+1)}}{3 m+1}\right] & \text { if } n=3\end{cases}
$$

Proof. We know that $G A_{5}(G)=\sum_{u v \in E(G)} \frac{2 \sqrt{S_{u} S_{v}}}{\left(S_{u}+S_{v}\right)}$

Case (1) If $n \geq 4$ :

$$
\begin{aligned}
G A_{5}\left(D_{n}^{(m)}\right)= & \left|E_{(4,4)}^{*}\right| \sum_{u v \in E_{(4,4)}^{*}(G)} \frac{2 \sqrt{S_{u} S_{v}}}{\left(S_{u}+S_{v}\right)}+\left|E_{(4,2 m+2)}^{*}\right| \sum_{u v \in E_{(4,2 m+2)}^{*}(G)} \frac{2 \sqrt{S_{u} S_{v}}}{\left(S_{u}+S_{v}\right)} \\
& +\left|E_{(2 m+2,4 m)}^{*}\right| \sum_{u v \in E_{(2 m+2,4 m)}^{*}(G)} \frac{2 \sqrt{S_{u} S_{v}}}{\left(S_{u}+S_{v}\right)}
\end{aligned}
$$

[From Table

2 and Figure 5]

Case (2) If $n=3$ :

$$
\begin{gathered}
=(n-4) m \frac{2 \sqrt{4 \cdot 4}}{4+4}+2 m \frac{2 \sqrt{4(2 m+2)}}{4+2 m+2}+2 m \frac{2 \sqrt{(2 m+2) 4 m}}{2 m+2+4 m} \\
=(n-4) m+\frac{4 m \sqrt{2(m+1)}}{3}+\frac{2 m \sqrt{2 m(m+1)}}{3 m+1} .
\end{gathered}
$$

$$
G A_{5}\left(D_{n}^{(m)}\right)=\left|E_{(2 m+2,2 m+2)}^{*}\right| \sum_{u v \in E_{(2 m+2,2 m+2)}^{*}(G)} \frac{2 \sqrt{S_{u} S_{v}}}{\left(S_{u}+S_{v}\right)}+\left|E_{(2 m+2,4 m)}^{*}\right| \sum_{u v \in E_{(2 m+2,4 m)}^{*}(G)} \frac{2 \sqrt{S_{u} S_{v}}}{\left(S_{u}+S_{v}\right)}
$$

Table 3. Edge partition based on degree sum of neighbors of end vertices of each edge.

\begin{tabular}{cc|}
\hline Edges of the type & Number of edges \\
\hline$E_{(2 m+2,2 m+2)}^{*}$ & $\mathrm{~m}$ \\
$E_{(2 m+2,2 m)}^{*}$ & $2 \mathrm{~m}$ \\
\hline
\end{tabular}


[From Table 3]

$$
\begin{gathered}
=m \frac{2 \sqrt{(2 m+2)(2 m+2)}}{2 m+2+2 m+2}+2 m \frac{2 \sqrt{(2 m+2) 4 m}}{2 m+2+4 m} \\
=m\left[1+\frac{4 \sqrt{2 m(m+1)}}{3 m+1}\right] .
\end{gathered}
$$

\section{Conclusion}

The problem of finding the general formula for $A B C$ index, $A B C_{4}$ index, Randic connectivity index, Sum connectivity index, $G A$ index and $G A_{5}$ index of Dutch Windmill Graph is solved here analytically without using computers.

\section{Acknowledgements}

The first author is also thankful to the University Grants Commission, Government of India for the financial support under the grant MRP(S)-0535/13-14/KAMY004/UGC-SWRO.

\section{Conflict of Interests}

The authors declare that there are no conflicts of interests regarding the publication of this paper.

\section{References}

[1] Estrada, E., Torres, L., Rodriguez, L. and Gutman, I. (1998) An Atom-Bond Connectivity Index: Modelling the Enthalpy of Formation of Alkanes. Indian Journal of Chemistry, 37A, 849-855.

[2] Chen, J., Liu, J. and Guo, X. (2012) Some Upper Bounds for the Atom-Bond Connectivity Index of Graphs. Applied Mathematics Letters, 25, 1077-1081. http://dx.doi.org/10.1016/j.aml.2012.03.021

[3] Chen, J. and Guo, X. (2012) The Atom-Bond Connectivity Index of Chemical Bicyclic Graphs. Applied MathematicsA Journal of Chinese Universities, 27, 243-252. http://dx.doi.org/10.1007/s11766-012-2756-4

[4] Xing, R., Zhou, B. and Dong, F. (2011) On Atom-Bond Connectivity Index of Connected Graphs. Discrete Applied Mathematics, 159, 1617-1630. http://dx.doi.org/10.1016/j.dam.2011.06.004

[5] Furtula, B., Gravoc, A. and Vukicevic, D. (2009) Atom-Bond Connectivity Index of Trees. Discrete Applied Mathematics, 157, 2828-2835. http://dx.doi.org/10.1016/j.dam.2009.03.004

[6] Gutman, I., Furtula, B. and Ivanovic, M. (2012) Notes on Trees with Minimal Atom-Bond Connectivity Index. MATCH Communications in Mathematical and in Computer Chemistry, 67, 467-482.

[7] Xing, R., Zhou, B. and Du, Z. (2010) Further Results on Atom-Bond Connectivity Index of Trees. Discrete Applied Mathematics, 157, 1536-1545. http://dx.doi.org/10.1016/j.dam.2010.05.015

[8] Xing, R. and Zhou, B. (2012) Extremal Trees with Fixed Degree Sequence for Atom-Bond Connectivity Index. FILOMAT, 26, 683-688. http://dx.doi.org/10.2298/FIL1204683X

[9] Ghorbani, M. and Hosseinzadeh, M.A. (2010) Computing $A B C_{4}$ Index of Nanostar Dendrimers. Optoelectronics and Advanced Materials: Rapid Communications, 4, 1419-1422.

[10] Farahani, M.R. (2013) Computing Fourth Atom-Bond Connectivity Index of V-Phenylenic Nanotubes and Nanotori. Acta Chimica Slovenica, 60, 429-432.

[11] Farahani, M.R. (2013) On the Fourth Atom-Bond Connectivity Index of Armchair Polyhex Nanotube. Proceedings of the Romanian Academy-Series B, 15, 3-6.

[12] Randic, M. (1975) On Characterization of Molecular Branching. Journal of the American Chemical Society, 97, 66096615. http://dx.doi.org/10.1021/ja00856a001

[13] Zhou, B. and Xing, R. (2011) On Atom-Bond Connectivity Index. Zeitschrift für Naturforschung, 66a, 61-66. http://dx.doi.org/10.5560/ZNA.2011.66a0061

[14] Zhou, B. and Trinajstic, N. (2009) On a Novel Connectivity Index. Journal of Mathematical Chemistry, 46, 1252-1270. http://dx.doi.org/10.1007/s10910-008-9515-Z

[15] Zhou, B. and Trinajstic, N. (2010) On General Sum-Connectivity Index. Journal of Mathematical Chemistry, 47, 210- 
218. http://dx.doi.org/10.1007/s10910-009-9542-4

[16] Vukicevic, D. and Furtula, B. (2009) Topological Index Based on the Ratios of Geometrical and Arithmetical Means of End-Vertex Degrees of Edges. Journal of Mathematical Chemistry, 46, 1369-1376. http://dx.doi.org/10.1007/s10910-009-9520-x

[17] Chen, S. and Liu, W. (2010) The Geometric-Arithemtic Index of Nanotubes. Journal of Computational and Theoretical Nanoscience, 7, 1993-1995. http://dx.doi.org/10.1166/jctn.2010.1573

[18] Das, K.C. and Trinajstic, N. (2010) Comparision between First Geometric-Arithmetic Index and Atom-Bond Connectivity Index. Chemical Physics Letters, 497, 149-151. http://dx.doi.org/10.1016/j.cplett.2010.07.097

[19] Xiao, L., Chen, S., Guo, Z. and Chen, Q. (2010) The Geometric-Arithmetic Index of Benzenoidsystems and Phenylenes. International Journal of Contemporary Mathematical Sciences, 5, 2225-2230.

[20] Graovac, A.. Ghorbani, M. and Hosseinzadeh, M.A. (2011) Computing Fifth Geometric-Arithmetic Index for Nanostar Dendrimers. Journal of Mahematical Nanoscience, 1, 33-42. 